\title{
The Construction of a Wind Turbine System with an EDLC for Hydrogen Production
}

\author{
Hironobu Kaneuchi Student Member (Tokyo University of Science, kaneuchi@mar.rikadai.jp) \\ Toshiaki Yachi Member (Tokyo University of Science, yachi@ee.kagu.tus.ac.jp) \\ Tatsuo Tani Member (Tokyo University of Science, SUWA, tani@rs.suwa.tus.ac.jp)
}

Keywords: wind turbine, EDLC, hydrogen

We have studied a new wind turbine system that uses an electric double layer capacitor (EDLC) as a method for effectively producing hydrogen. In this paper, the method to determine the system configuration that would effectively supply the electric energy to the hydrogen generator was examined using numerical analysis. This system consisted of the EDLC and the electric charge and discharge control device (MPPT charger and DC/DC converter) inserted between the wind turbine and the hydrogen generator (Fig. 1). By buffering against changes in power generation due to fluctuations in wind speed, the system provided a constant source of electric power to the hydrogen generator. We used a wind turbine generator with a rated output of $500 \mathrm{~W}$ and an EDLC with 2 or more cells connected in series with a rated voltage of $2.7 \mathrm{~V}$, a static electric capacitance of $1240 \mathrm{~F}$, and an internal resistance of $1.8 \mathrm{~m} \Omega$. For the wind simulation model, the data of Miyako Island ( $4.7 \mathrm{~m} / \mathrm{s}$ average wind speed) was used in the analysis.

The electric power supplied to the hydrogen generator was influenced by the relationship between the amount of electric power produced by the wind turbine and the capacity of the hydrogen generator. The dependence of the hydrogen generator load capacity on wind speed was examined. In this analysis, it was assumed that the EDLC had been removed from the system. As well, it was assumed that if the DC/DC converter output was greater than the hydrogen generator load capacity, the output was equal to the rated power. Let us represent the surplus electric energy as $W_{1}$, when the DC/DC converter output is larger than the hydrogen generator load capacity. Similarly, the deficient electric energy is represented by $W_{2}$, when DC/DC output is smaller than the hydrogen generator load capacity. Under these conditions, according to the change of the hydrogen generator load capacity, $W_{1}$ and $W_{2}$ change respectively (Fig. 2). With an increase in the hydrogen generator load capacity, the surplus electric energy, $W_{1}$, decreased, while the deficient electric energy, $W_{2}$, increased. Furthermore, it should be noted that $W_{1} \cong W_{2}$ when the hydrogen generator load capacity was $144.0 \mathrm{~W}$, at which the electric energy supplied to the hydrogen generator, $W_{s}$, is at its maximum value. Since the surplus electric energy, $W_{1}$, is equivalent to the electric energy charge in the EDLC, and since the deficient electric energy, $W_{2}$, is equivalent to the electric discharge

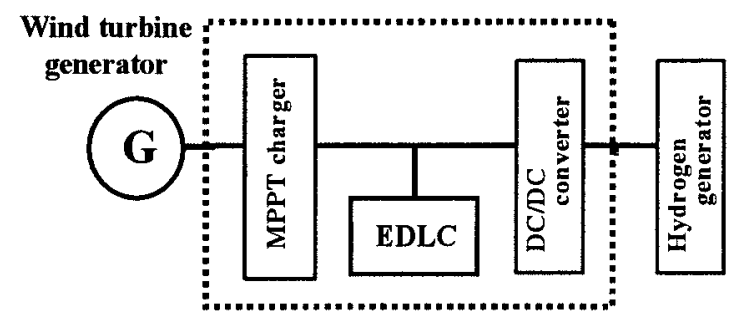

Fig. 1. System configuration

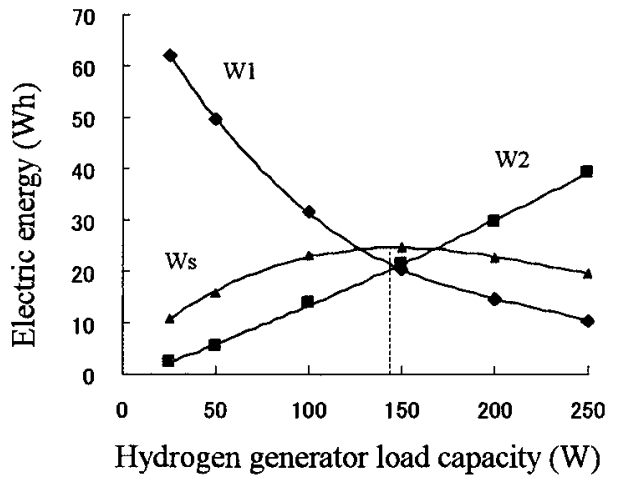

Fig. 2. Electric energy depended on hydrogen generator load capacity

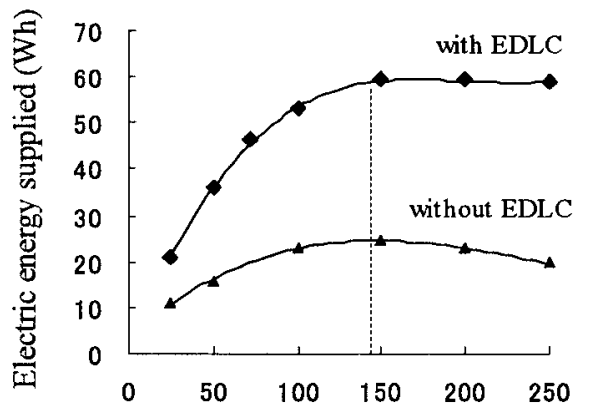

Hydrogen generator load capacity (W)

Fig. 3. Relationship between the hydrogen generator load capacity and the supply of electric energy

energy from the EDLC, these results suggest that if an EDLC is connected, then the electric charge and discharge of an EDLC would be balanced when $W_{1} \cong W_{2}$. Consequently, the relation, $W_{1} \cong W_{2}$, is considered to be the governing condition for electric energy to be effectively supplied to the hydrogen generator. Then, the EDLC capacity can be reduced without significantly decreasing the supply of electric energy to the hydrogen generator.

The relationship between the electric energy supply and hydrogen generator load capacity is shown for a system consisting of EDLC with a proper capacity (Fig. 3). The supply of electric energy tended to increase with an increase in the hydrogen generator load capacity. However, the electric energy supplied became constant once the electric energy supply reached the proper capacity of the hydrogen generator shown with the dotted line, which occurs when the EDLC is disconnected. When no further increase in the electric energy supplied could be expected, the present method to determine the appropriate configuration was confirmed to be correct. 


\title{
The Construction of a Wind Turbine System with an EDLC for Hydrogen Production
}

\author{
Hironobu Kaneuchi* Student Member \\ Toshiaki Yachi* Member \\ Tatsuo Tani** Member
}

\begin{abstract}
We studied a new wind turbine system that uses an electric double layer capacitor (EDLC) as a method of effectively producing hydrogen from natural energy. Before this system can be installed and its effects considered, an outline design of the system based on the wind conditions in the installation region must be performed, and the system scale and the obtained amount of the electric power must be estimated. In this paper, the method to determine the system configuration that would effectively supply the electric energy to the hydrogen generator was examined using numerical analysis. The configuration obtained from this analysis determined the required capacity of the hydrogen generator for the amount of power generation that was needed, as well as the conditions required in order for the EDLC to effectively buffer any changes. When a system is constructed using this configuration, the electric charging and discharging of the EDLC are balanced, and it is possible to effectively supply electric energy to the hydrogen generator. When a wind turbine generator with a rated power of $500 \mathrm{~W}$ was operated under the wind conditions on Miyako Island, the appropriate capacity of the hydrogen generator was $144 \mathrm{~W}$, and the corresponding EDLC capacity was $11.3 \mathrm{Wh}$.
\end{abstract}

Keywords: wind turbine, EDLC, hydrogen

\section{Introduction}

Recently, wind power that uses a natural power source as an independent power supply has attracted attention. In order to use wind power as an independent power supply, it is necessary to equalize both the change in the amount of power generated due to the intermittent nature of wind and the supply of electric energy to the load ${ }^{(1)}$. One such possible method is to use wind power to produce hydrogen, which is then later used to produce electric energy via a hydrogen fuel cell $^{(2)}$. In order to produce hydrogen efficiently from wind power, it has been suggested that a system with an electric double layer capacitor (EDLC) can be used as the electric power buffer medium ${ }^{(2)-(4)}$. Since the secondary battery's life cycle is important, the frequent changes in the generation of power, which is caused by the electrical charge and discharge cycles, needs to be minimised. Moreover, attention is needed to avoid any overcharge or any electric overdischarge due to the irregular electrical charge and discharge pattern. Since an EDLC has low degradation by the electric charge and discharge cycles, as well as accurate control compared with existing chemical secondary batteries, it is a useful medium to buffer changes in the amount of power generated ${ }^{(5)}$. However, since an EDLC is expensive compared with other storage batteries and has a low energy density, it is necessary to determine the appropriate capacity. Before this system can be installed and its effects considered, an outline design of the system based on the wind conditions in the installation

\footnotetext{
* Tokyo University of Science

1-3, Kagurazaka, Shinjyuku-ku, Tokyo 162-8601

** Tokyo University of Science, Suwa

1-5000, Toyohira, Chino 391-0292
}

region must be performed, and the system scale and the obtained amount of the electric power must be estimated. Thus, this study sought to determine, using numerical analysis, a method that would create an appropriate system configuration that would supply electric power to the hydrogen generator.

\section{Analysis of the Model}

2.1 System Configuration The structure of the hydrogen producing system used in this simulation is shown in Fig. 1. This system consisted of the EDLC, as well as the electric charge and discharge control device (MPPT charger and $\mathrm{DC} / \mathrm{DC}$ converter) inserted between the wind turbine and the hydrogen generator. By buffering against changes in power generation due to fluctuations in wind speed, the system provided a constant source of electric power to the hydrogen generator. A Windseeker503 (rated output $500 \mathrm{~W}$ ) was used as a sample small wind turbine generator.

The output characteristics of the Windseeker503 are shown in Fig. $2^{(6)}$. An EDLC, with 2 or more cells connected in series with a rated voltage of $2.7 \mathrm{~V}$, a static electric capacitance of $1240 \mathrm{~F}$, and an internal resistance of $1.8 \mathrm{~m} \Omega$,was used ${ }^{(5)}$.

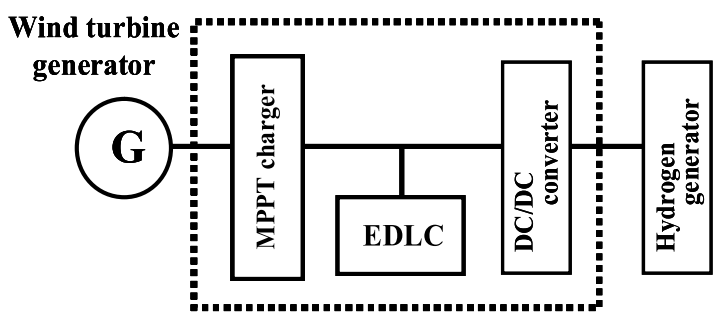

Fig. 1. System configration 


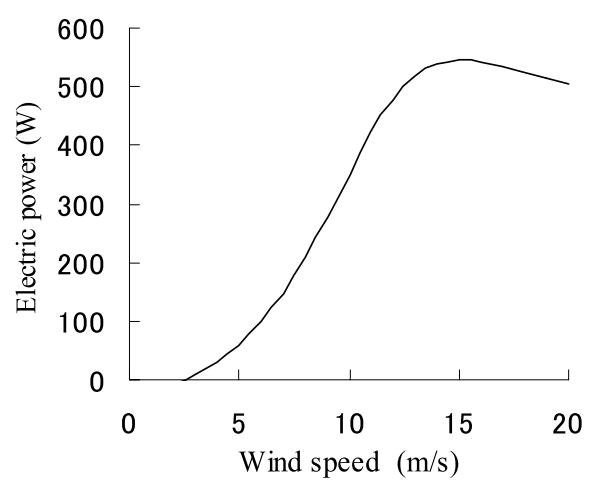

Fig. 2. The output characteristics of the Windseeker503

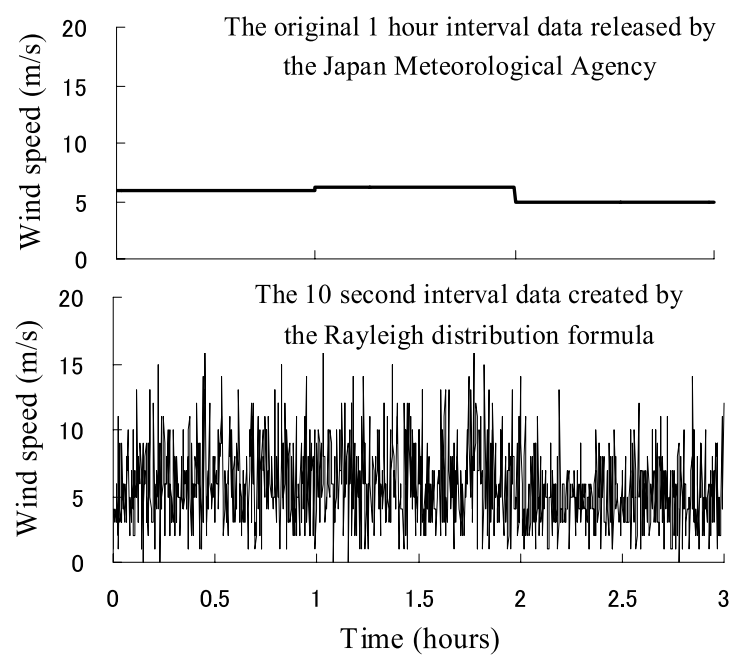

Fig. 3. Wind speed model

To prevent no uniformity of the voltage distribution between cells when the cell is connected in series, the control circuit is built into each cell ${ }^{(5)}$. The power consumption in this circuit is not considered because it is very small compared with the electric charge and discharge. Moreover, the self-electric discharge of the EDLC was not considered and the electric capacitance and internal resistance of the cell were assumed to be constant regardless of the operating state.

2.2 Creation of Detailed Wind Speed Data The wind simulation used 1 month's wind speed data, which reflected the annual average wind speed. Since more detailed wind speed data was needed for this analysis, the original data with a 1 hour interval as released by the Japan Meteorological Agency ${ }^{(7)}$ was converted into 10 second interval data using a Rayleigh distribution ${ }^{(8)}$ and random numbers. The Rayleigh distribution formula is given in Eq. (1).

$$
f(v)=\frac{\pi}{2} \cdot \frac{v}{V^{2}} \exp \left\{-\frac{\pi}{4}\left(\frac{v}{V}\right)^{2}\right\}
$$

$f(v)$ : probability of observing a given wind speed $v$ : wind speed

$V$ : average wind speed

The Rayleigh distribution gives the probability of observing a given wind speed based on a given average wind speed. Sample data to which the Rayleigh distribution was applied is shown in Fig. 3. For the wind simulation model, the data from Miyako Island ( $4.7 \mathrm{~m} / \mathrm{s}$ average wind speed, Fig. 4) was used in the analysis.

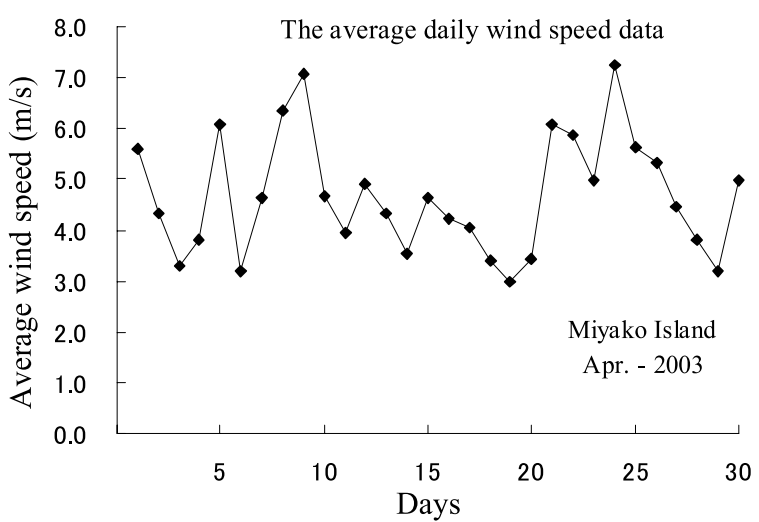

Fig. 4. The wind speed data from Miyako Island

\section{System Control}

3.1 Electric Power Control The system control flow chart is shown in Fig. 5. The electric power obtained with the wind simulation data and the basic output characteristics of the wind turbine generator enter into the system through the MPPT charger. The MPPT charger output is given priority over the EDLC output supplied to the DC/DC converter.

As shown in the model in Fig. 6, when the wind is strong enough to generate a large amount of power, the MPPT charger outputs the electric energy to the DC/DC converter, while any surplus electric energy is simultaneously used to charge the EDLC. However, when the wind weakens and power generation falls, electric energy is discharged from the EDLC to compensate for the lack of electric energy output from the MPPT charger. Electric discharge is possible if the EDLC terminal voltage reaches the control voltage. When the EDLC terminal voltage reaches the rated voltage, charging stops, and all the surplus electric energy is considered to be lost. The electric energy passed to the DC/DC converter is then supplied to the hydrogen generator after being converted into the rated power.

3.2 Electric Charge Method of EDLC It is necessary to consider the efficiency of EDLC charging. When a constant voltage (voltage $E$ ) is used, the charging current, $i$, will generally be given by Eq. (2):

$$
i=\frac{E}{R} \exp \left(-\frac{t}{C R}\right)
$$

where $R$ is the internal resistance, $C$ is the EDLC capacitance, and $t$ is the elapsed time. As a result, a large initial charging current will be created, leading to a large loss of power. On the other hand, in the case of a constantly charging current, it is possible to mitigate the energy loss due to internal resistance by keeping the charging current small and constant. However, it is difficult for electric power production to efficiently keep the charging current constant with an unstable power supply like a wind turbine generator. Therefore, in a system where the electric power generated is constantly changing, the change in source current charge can be used to control the charging current, $I$, and the output to the EDLC by using a switch converter operation.

The electric energy, $W_{C i n}$, inputted into the EDLC through a MPPT charger, along with the generated power, $P_{G}$, using a wind turbine generator, is given by Eq. (3), where $P_{M}$ is 


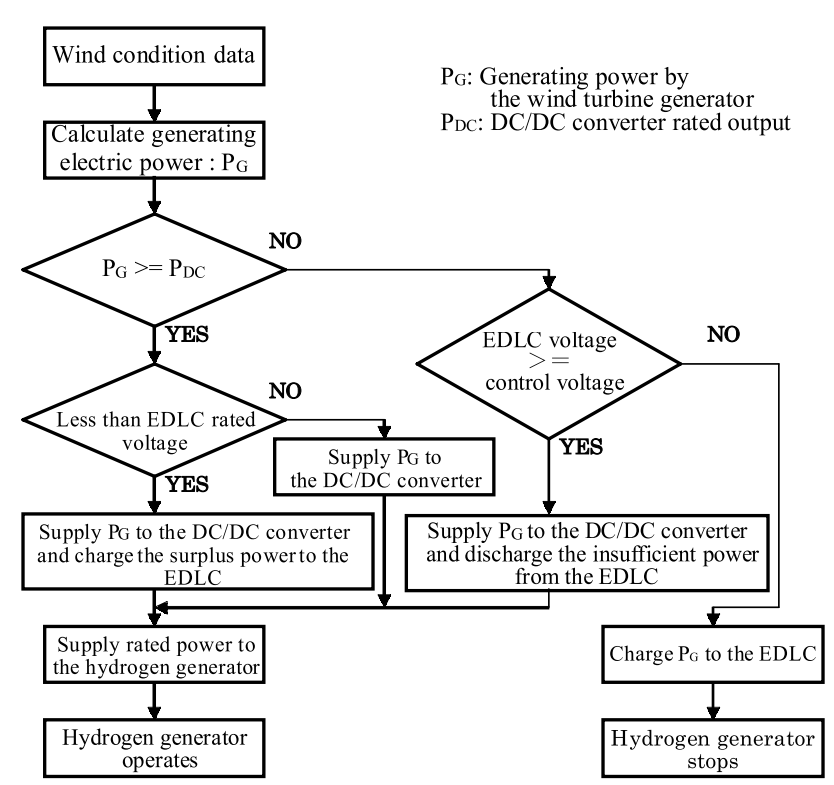

Fig. 5. Flow chart of the system control
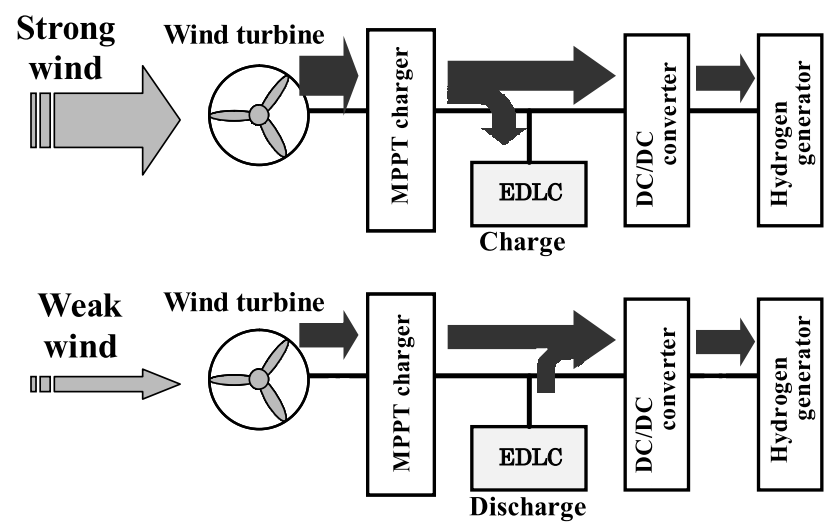

Fig. 6. System control schematic diagrams

the power consumption of the MPPT charger, $P_{H}$ is the load capacity of a hydrogen generator and $\eta_{D C}$ is the conversion efficiency of the DC/DC converter. Furthermore, $W_{C i n}$ can also be expressed using Eq. (4), where $W_{c}$ is the charged energy in the EDLC and $W_{R}$ is the energy loss due to internal resistance.

$$
\begin{aligned}
& W_{C i n}=\left\{\left(P_{G}-P_{M}\right)-\frac{P_{H}}{\eta_{D C}}\right\} \cdot t \\
& W_{C i n}=W_{C}+W_{R} \ldots \ldots \ldots \ldots \ldots
\end{aligned}
$$

As well, Eqs. (5) and (6) give the $P_{c}$ and $P_{R}$ after $t$-seconds, where $I$ is the charging current and $V_{c}$ is the EDLC voltage. Then the EDLC voltage can be expressed using Eq. (7), setting the EDLC electric charge at $t=0$ to $Q_{0}$.

$$
\begin{aligned}
& W_{c}=\int_{0}^{t} V_{c} \cdot I d t \ldots \ldots \ldots \ldots \ldots \ldots \ldots \ldots \ldots \ldots \ldots \ldots \ldots \ldots \\
& W_{R}=\int_{0}^{t} I^{2} R d t \\
& V_{c}=\frac{Q_{0}+I t}{C} .
\end{aligned}
$$

Eq. (8) is derived from the above equations, and allows the charging current in the EDLC to be determined.

$$
\left\{\left(P_{G}-P_{M}\right)-\frac{P_{H}}{\eta_{D C}}\right\} \cdot t=\frac{I t}{C} Q_{0}+\frac{(I t)^{2}}{2 C}+I^{2} R t \cdots
$$

\section{Analysis Procedure}

The electric power supplied to the hydrogen generator influences the relationship between the amount of electric power produced by the wind turbine and the load capacity of the hydrogen generator. The dependence of the hydrogen generator load capacity on wind speed was examined first. In this way, the optimal load capacity of the hydrogen generator for electric power production was determined.

Next, the influence of charge efficiency and electric discharge depth of the EDLC on the buffer medium was examined. From these results, the capacity of the EDLC to efficiently supply electric power to the hydrogen generator, as well as a suitable EDLC capacity, was found.

Finally, the resulting system configuration was evaluated.

\section{Results of Analysis}

5.1 Dependence of Hydrogen Generator Load Capacity on Wind Speed In this analysis, it was assumed that the EDLC had been removed from the system. It was assumed that if the DC/DC converter output, $P_{0}$, was greater than the hydrogen generator's load capacity, the output was equal to the rated power, and if the DC/DC output was less than the load capacity, then no power was outputted. $P_{0}$ is given by Eq. (9).

$$
P_{0}=\left(P_{G}-P_{M}\right) \times \eta_{D C}
$$

The amount of power generated, $P_{G}$, was calculated from the basic output characteristics of the wind turbine and the wind speed. The MPPT power consumption, $P_{M}$, was assumed to be $10 \mathrm{~W}$, while the DC/DC converter conversion efficiency, $\eta_{D C}$, was assumed to be $80 \%$.

The relationship between the hydrogen generator load capacity and the hourly average electric energy supplied under the wind conditions on Miyako Island for 1 month is shown in Fig. 7. It would appear that the amount of electric power supplied varied greatly due to changes in the hydrogen generator load capacity, and, thus, depended on the hydrogen generator load capacity. Although the wind turbine's average hourly electric energy generation was $85.5 \mathrm{Wh}$ for the given wind simulation, the electric energy supplied to the hydrogen generator peaked at $24.4 \mathrm{Wh}$, when the hydrogen generator load capacity reached $144.0 \mathrm{~W}$.

If we let the surplus electric energy be $W_{1}$ when the DC/DC converter output is larger than the hydrogen generator load capacity, then $W_{1}$ is given by Eq. (10). Similarly, if the deficient electric energy is represented by $W_{2}$, when DC/DC output is smaller than the hydrogen generator load capacity, then $W_{2}$ is given by Eq. (11).

$$
\begin{array}{ll}
W_{1}=\left(P_{0}-P_{H}\right) \cdot t & \left(P_{0}>P_{H}\right) \\
W_{2}=\left(P_{H}-P_{0}\right) \cdot t & \left(P_{H}>P_{0}\right)
\end{array}
$$

Figure 8 is a pattern plot showing the electric energy supplied to the hydrogen generator, $W_{s}$, the surplus electric energy, $W_{1}$, and the deficient electric energy, $W_{2}$. Figure 7 shows the change in the electric energy supplied to the hydrogen generator given the variation in capacity shown in Fig. 8. 


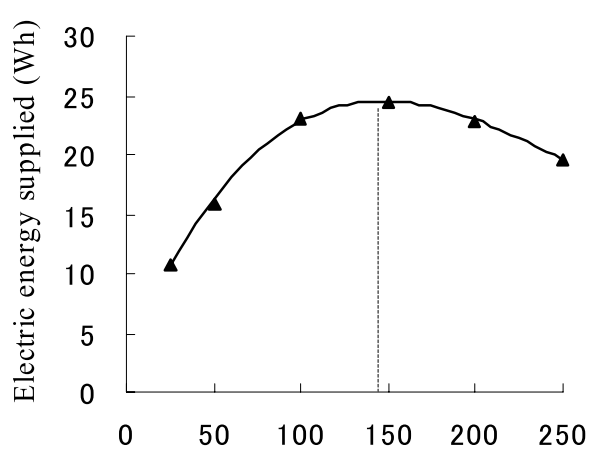

Hydrogen generator load capacity (W)

Fig. 7. Supplied energy without EDLC depends on the hydrogen generator load capacity for the wind conditions on Miyako Island

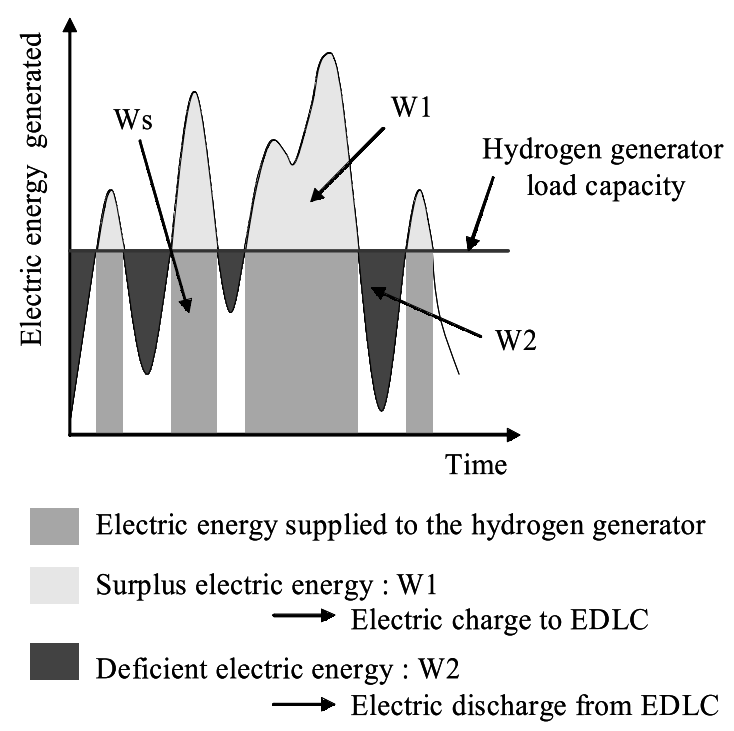

Fig. 8. The pattern plot of generating electric energy

In this case, the change in $W_{1}$ and $W_{2}$ depends on the hydrogen generator load capacity.

The relation of the average hourly amount of the surplus electric energy, $W_{1}$, the deficient electric energy, $W_{2}$, and $W_{1}+W_{2}$ by the hydrogen generator load capacity is shown in Fig. 9. With an increase in the hydrogen generator load capacity, the surplus electric energy, $W_{1}$, decreased, while the deficient electric energy, $W_{2}$, increased. Furthermore, it should be noted that when $W_{1} \cong W_{2}$ the hydrogen generator load capacity was $144.0 \mathrm{~W}$, at which point the electric energy supplied to the hydrogen generator was at its maximum value (Fig. 7). Since the surplus electric energy, $W_{1}$, is equivalent to the electric energy charge in the EDLC, and the deficient electric energy, $W_{2}$, is equivalent to the electric discharge energy from the EDLC, these results suggest that if an EDLC is connected, then the electric charge and discharge of an EDLC would be balanced when $W_{1} \cong W_{2}$. Thus, setting the hydrogen generator load capacity to the point where $W_{1} \cong W_{2}$ in the system allows for efficient operation of the EDLC. In addition, the value of $W_{1}+W_{2}$ minimum can reduce the capacity of EDLC in the situation of. $W_{1} \cong W_{2}$. Consequently, the relationship, $W_{1} \cong W_{2}$, is considered to be the governing condition for electric energy to be effectively supplied to a hydrogen generator. Therefore, the proper

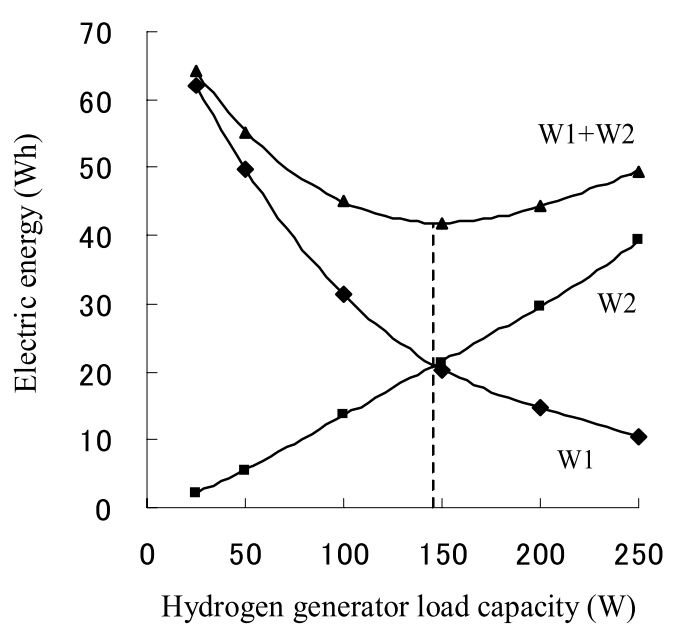

Fig. 9. Dependence of $W_{1}$ and $W_{2}$ on the hydrogen generator load capacity for the wind conditions on Miyako Island

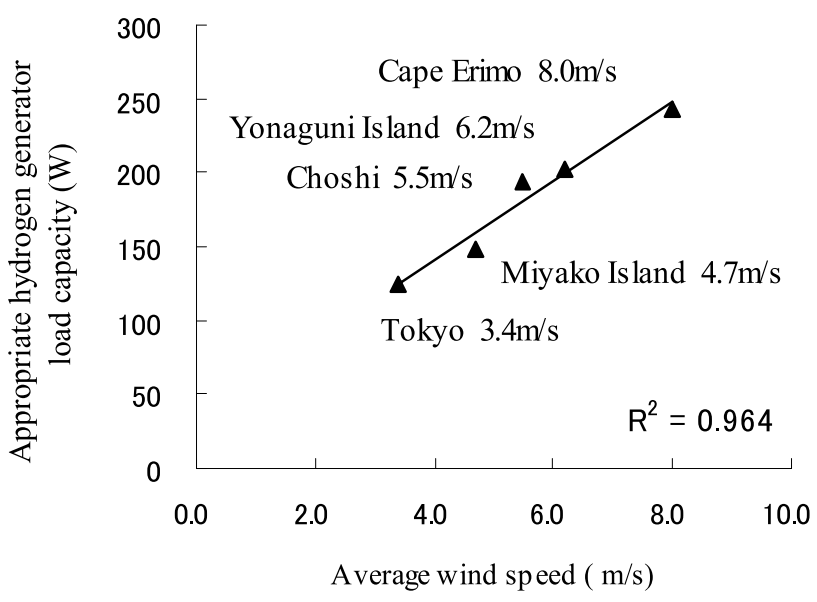

Fig. 10. The dependence of hydrogen generator load capacity on the average wind speed

hydrogen generator load capacity in the system for the wind conditions on Miyako Island was assumed to be $144 \mathrm{~W}$ for this analysis.

The amount of the electric power developed from the wind turbine generator depends on the wind condition. Thus, the dependence of hydrogen generator load capacity on the wind condition was investigated. The wind conditions on Cape Erimo in Hokkaido (average wind speed of $8.0 \mathrm{~m} / \mathrm{s}$ ), Yonaguni island (average wind speed of $6.2 \mathrm{~m} / \mathrm{s}$ ) and Choshi (average wind speed of $5.5 \mathrm{~m} / \mathrm{s}$ ) where the wind speeds are stronger than on Miyako Island, as well as data from Tokyo (average wind speed of $3.4 \mathrm{~m} / \mathrm{s}$ ) where the wind speed is weaker than on Miyako Island, were studied next. The load capacity for the proper hydrogen generator in each wind situation was examined by using an analysis method similar to that shown above. The proper hydrogen generator load capacity was determined to be $258 \mathrm{~W}$ for Cape Erimo, $203 \mathrm{~W}$ for Yonaguni Island, $193 \mathrm{~W}$ for Choshi and $125 \mathrm{~W}$ for Tokyo. Fig. 10 shows the relationship between the average wind speed and the proper hydrogen generation capacity for each wind situation. A probable reason that explains the difference seen in the appropriate hydrogen generator capacities was that the amount of power generated depended on the changing 
instantaneous wind speeds, even if the average wind speed was the same. The appropriate hydrogen generator load capacity used for the system can be approximated by using average wind speed data for an arbitrarily chosen system installation region based on the relationship between the average wind speed and the capacity of the appropriate hydrogen generator as shown in Fig. 10. The coefficients of determination, $R^{2}$, that showed the level of the data correlation was 0.964 . In the wind speed data with a large change element, it was thought that the correlation was high.

5.2 Influence of the EDLC Control Voltage In order to use the limited EDLC capacity effectively, it is necessary to set the EDLC's electric discharge depth at a deep level. It is well known that the lifetime of the EDLC isn't influenced unlike other storage batteries even if the electrical discharge depth is set deeply. On the other hand, the lifetime decreases by frequent float charge that puts a heavy strain on the EDLC cell when the situation continues, in which the electrical discharge depth of the EDLC is set extremely shallowly and the full charge is always maintained ${ }^{(5)}$. However, in this system that stored electric energy is constantly converted into hydrogen, the period in the state of the full charge is short even if the electrical discharge depth is comparatively shallow since the electric charge and discharge of the EDLC is frequently repeated. Consequently, it is considered that the lifetime of the EDLC used for this system is not influenced so much even when the electrical discharge depth is comparatively shallow. Therefore, the electrical discharge depth of the EDLC can be arbitrarily set without considering the lifetime unlike other storage batteries. The depth of electric discharge depends on the EDLC control voltage that makes electric discharge possible when the EDLC voltage is greater than the control voltage. In order to investigate the effects of changing the control voltage on the electric energy buffer operation of the EDLC, the relationship of charge efficiency for each EDLC voltage was calculated. The EDLC charge efficiency $\eta i$ is given by Eq. (12), which is derived from Eqs. (5) to (7), where $t$ is the charging time, and $Q_{0}$ is the initial charge calculated by the EDLC terminal voltage.

$$
\eta i=\frac{W_{c}}{W_{c}+W_{R}}=\frac{Q_{0}+I t / 2}{Q_{0}+I t / 2+C R I} \cdots \cdots \cdots \cdots
$$

Figure 11 shows the relationship between the EDLC terminal voltage for each charging current and the charging efficiency calculated by the Eq. (12) when $t=1 \mathrm{~s}$. The EDLC terminal voltage is standardized to the rated voltage. In the area where EDLC terminal voltage is low, that is, if the EDLC can only hold a small amount of charge, charge efficiency falls greatly. Moreover, although efficiency tended to fall when the charging current increased, charge efficiency improved as the EDLC terminal voltage increased. Therefore, a greater electric discharge depth implies a lower control voltage setting. It is anticipated that the charge and discharge loss increases with the reduction of charge and discharge efficiency. On the other hand, although charge efficiency improved when the control voltage was set higher, electric discharge depth decreased. Consequently, the surplus electric energy lost increased, since the surplus energy could not be used to charge an already fully charged EDLC.

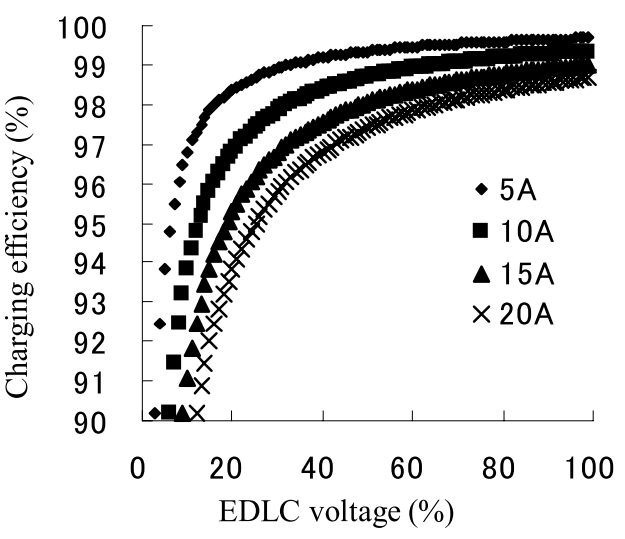

Fig. 11. Relationship between EDLC voltage and charging efficiency in an EDLC

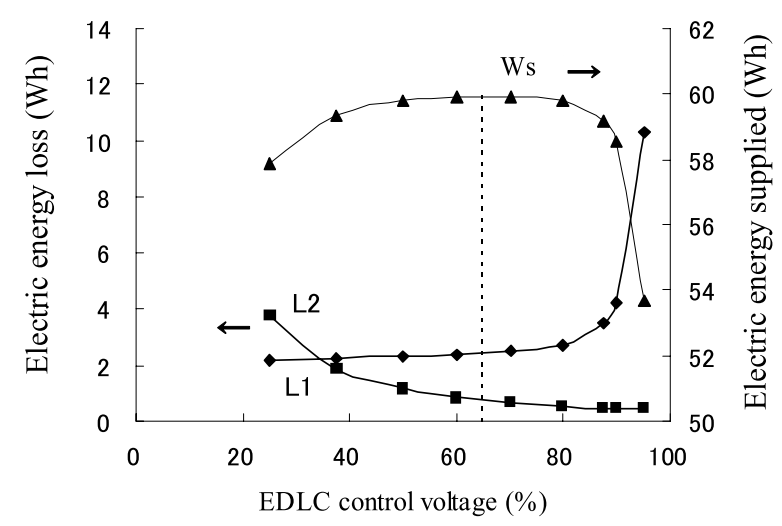

Fig. 12. Dependence of electric energy supplied and lost on the EDLC control voltage for a 12.56 Wh capacity

Figure 12 shows the dependence of the average hourly surplus electric energy loss, $L_{1}$, charge and discharge loss, $L_{2}$, and the mean hourly value of the electric energy supplied to the hydrogen generator, $W_{s}$, for a given EDLC control voltage for the wind conditions on Miyako Island during 1 month. An EDLC capacity of $12.56 \mathrm{Wh}$ was arbitrarily chosen as a sample. Then the EDLC control voltage was standardized to the rated voltage. This analysis produced similar results to those predicted above. The electric energy supplied to a hydrogen generator depended on the EDLC control voltage. Therefore, this implies that the electric energy supplied can be improved by properly adjusting the EDLC control voltage.

Figure 13 shows the relationship between the EDLC control voltage and the hourly average electric energy supplied to the hydrogen generator, assuming that the EDLC capacity is a parameter. When the EDLC capacity is relatively large, the influence of the control voltage on the electric energy supplied to the hydrogen generator is small. On the other hand, the effect increases as the EDLC capacity decreases.

This factor is associated with a change in the electric energy loss shown in Fig. 14, when it is assumed that EDLC capacity is a parameter. The average hourly surplus electric energy loss, $L_{1}$, and electric charge and discharge loss, $L_{2}$, combine to form the total loss. The surplus electric energy loss means the electric energy that cannot be charged when the EDLC becomes fully charged. Though the surplus electric energy loss tended to increase with an increase in the 
EDLC control voltage, the increase was also observed when the EDLC capacity decreases. This phenomenon implies that the actual amount that can be accumulated may depend on the EDLC capacity even if the control voltage is the same. Therefore, the loss increases with smaller EDLC capacity. On the other hand, the electrical charge and discharge loss increases with a smaller capacity. This phenomenon causes larger current to flow, and this loss may increase even if the electric energy is charged, because the rated voltage becomes smaller

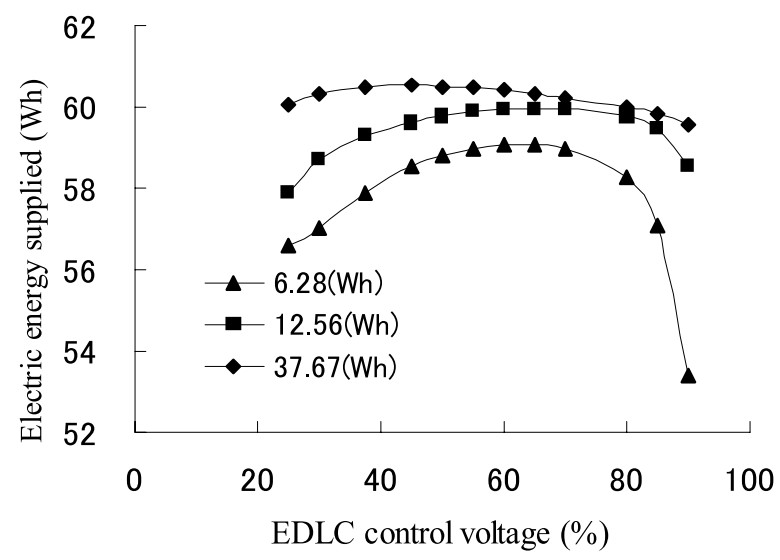

Fig. 13. Dependence of electric energy supplied on EDLC control voltage for the wind conditions on Miyako Island

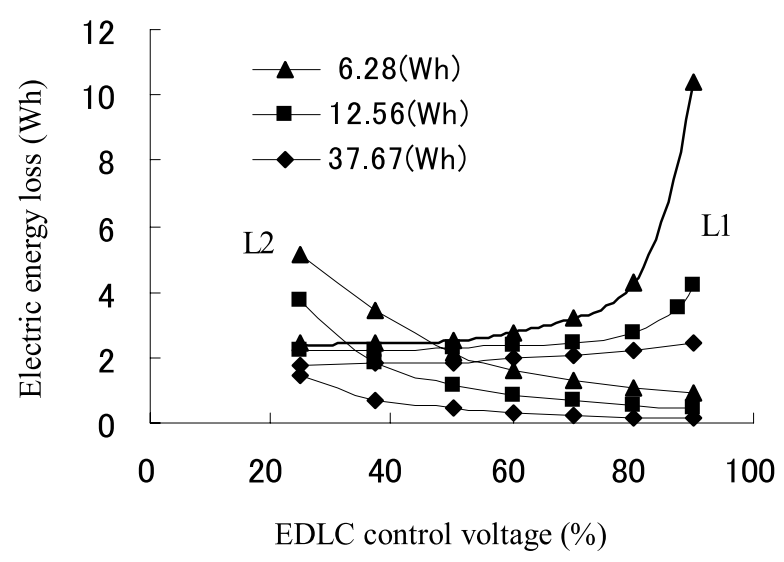

Fig. 14. Dependence of electric energy loss on EDLC control voltage for the wind conditions on Miyako Island

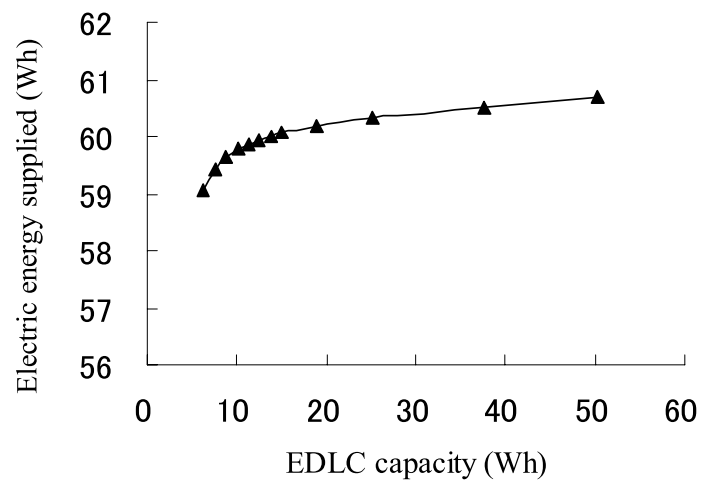

Fig. 15. Dependence of electric energy supplied on the EDLC capacity for the wind conditions on Miyako Island as the EDLC capacity become smaller. Therefore, based on these results, in order to avoid a small EDLC capacity in the system, setting a proper control voltage should be taken into consideration.

5.3 Proper EDLC Capacity in the System Figure 15 shows the relationship between the hourly average electric energy supplied and the EDLC capacity for the conditions on Miyako Island, when the proper control voltage is set so that the electric energy supplied to the hydrogen generator is maximized for each EDLC capacity. Although the electric energy supplied to the hydrogen generator decreased slightly with a decrease in EDLC capacity, it was very small compared with the decrease in the EDLC capacity. This result depends greatly on the balance between the amount of power generation and the hydrogen generator load capacity. It seems that a balance has been obtained when the proper capacity is selected properly. Thus, this system always converts the electric power generated into hydrogen and the balanced system produces a situation where the surplus electric energy is discharged into the EDLC. It was shown that the EDLC acts as the buffer medium that repeats the electric energy charge and discharge operation in such a comparatively short time that it functions even with a very small capacity. An EDLC capacity of $11.3 \mathrm{Wh}$, which corresponds to the inflection point in the amount of change, was considered to be the proper capacity. In this case, the control voltage was $69 \%$ of the EDLC rated voltage. Moreover, the amount of electric energy supplied to the hydrogen generator was $59.9 \mathrm{Wh}$, and the supply ratio of efficiency over power generation of electric energy was $69.8 \%$.

As well, for the wind conditions on Cape Erimo and Tokyo, the relationship between the electric energy supplied and the EDLC capacity had similar tendencies to those observed for the data based on the wind conditions on Miyako Island. This shows that the system also works at small EDLC capacity. Based on these results, an EDLC capacity of $50.2 \mathrm{Wh}$ for Cape Erimo and 8.79 Wh for Tokyo were considered to be the appropriate capacities.

\section{Confirmation of Construction Method}

This system was evaluated based on the results that have been obtained so far. In Fig. 16, the relationship between the

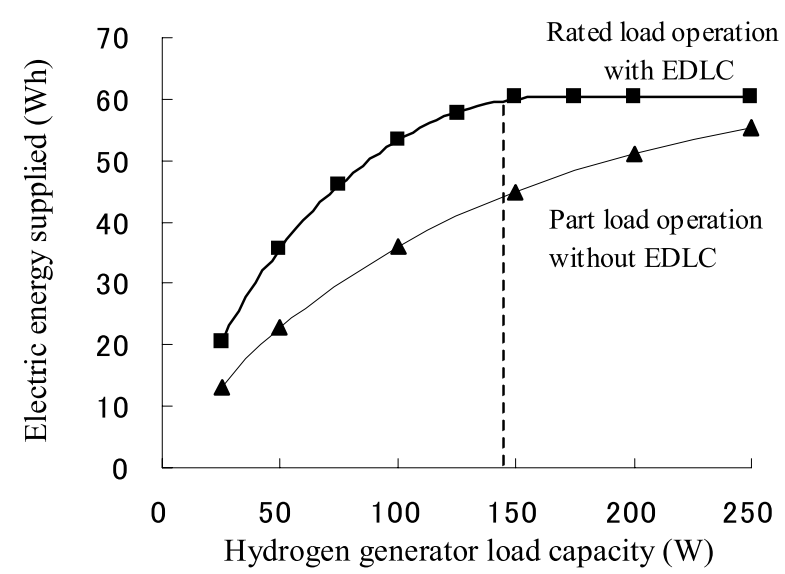

Fig. 16. Relationship between the hydrogen generator load capacity and the supply of electric energy for the Miyako Island wind situation 


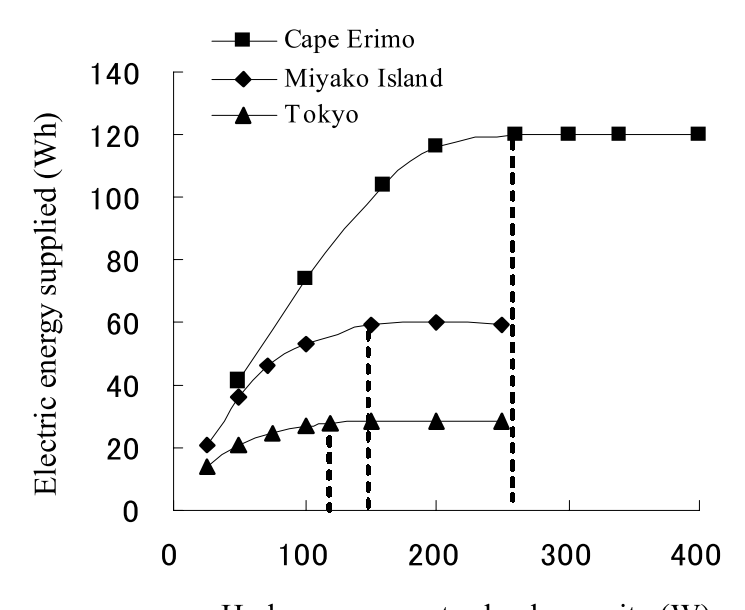

Hydrogen generator load capacity (W)

Fig. 17. The dependence of the electric energy supplied on the hydrogen generator load capacity

hourly average electric energy supply and hydrogen generator load capacity in the wind situation of Miyako Island for one month is shown in both the cases of a part load operation without connecting EDLC and a rated load operation with connecting EDLC with a capacity of $11.3 \mathrm{Wh}$.

In the former case, the supply of electric energy tended to increase with an increase in the hydrogen generator load capacity. Therefore, the electric energy supplied can be improved by making the hydrogen generator load capacity larger. However, an increase in the load capacity leads to the decrease in the utilized capacity of hydrogen generator because the hourly increase of the electric energy supplied tends to became gradually small with the increase in the load capacity. In the latter case, the electric energy supplied became constant when the proper load capacity of the hydrogen generator is more than $144 \mathrm{~W}$ which was led on the analytical condition assumed that "if the DC/DC converter output was greater than the hydrogen generator load capacity, the output was equal to the rated power, and if the DC/DC converter output was less than the load capacity, then no power was outputted". It is shown that this load capacity of $144 \mathrm{~W}$ is proper from the viewpoint of the electric energy supplied and the utilized capacity of hydrogen generator. Moreover, while the hourly average electric energy supplied to the hydrogen generator without connecting EDLC was $43.8 \mathrm{Wh}$, an hourly increase of $14.0 \mathrm{Wh}$ in the electric energy supplied was expected when the EDLC with a capacity of $11.3 \mathrm{Wh}$ was connected. At this point, the amount of electric energy supplied was 1.4 times as large as when there was no EDLC. Thus, by using EDLC for the system, the rated power is supplied to the hydrogen generator and the effect of improving the utilized capacity of the hydrogen generator is expected. Then, it is important for composing the system to lead the hydrogen generator load capacity in the relation that the surplus electric energy is equal to the deficient electric energy according to this analytical condition.

Furthermore, the relationships between the hydrogen generator load capacity and the average hourly electric energy supplied for systems based on the 1-month wind conditions in Miyako Island, Cape Erimo, and Tokyo, when the proper EDLC capacity was connected are shown in Fig. 17. It can be seen that the electric energy supplied tended to increase with an increase in the hydrogen generator load capacity for Cape Erimo, Tokyo, and Miyako Island. The electric energy became constant when the proper capacity for each hydrogen generator was reached, which is shown as a dotted line. This suggests that the results obtained were similar to those on Miyako Island. Thus, the system configuration can be considered to be appropriate for the given conditions. Therefore, the system design that was analyzed in this paper can be used to create a suitable system for locations with different wind conditions.

\section{Conclusion}

A method to determine the appropriate configuration of a wind turbine generation system for the production of hydrogen was examined. The following results were obtained:

(1) In a system that is constantly converting stored electric energy into hydrogen, there exists an appropriate capacity for the hydrogen generator that corresponds to the amount of the electric energy generated. When the electric charging and discharging of the EDLC are balanced by setting the correct value for the load capacity of the hydrogen generator, the buffer ability of the EDLC improves.

(2) The energy lost due to the buffer operation of the EDLC is minimized by properly setting the control voltage that determines the depth of the EDLC electrical discharge.

(3) By selecting the correct load capacity of the hydrogen generator and the correct EDLC control voltage, the EDLC capacity can be reduced without significantly decreasing the supply of electric energy to the hydrogen generator.

(4) The procedure to determine the system configuration is as follows. First, the appropriate load capacity of the hydrogen generator for a desired power generation level is selected. Next, the electric discharge depth suitable for the EDLC capacity is determined. Finally, an appropriate EDLC capacity derived from the relationship between EDLC capacity and the amount of electric energy needed to supply a hydrogen generator are found. Thus, this system configuration can be assumed to deliver an effective supply of electric energy to the hydrogen generator.

(Manuscript received July 25, 2005, revised June 8, 2006)

\section{References}

( 1 ) NEDO: "Storage battery juxtaposition wind power introduction possibility investigation", NEDO-NP-0004 (in Japanese) (2000)

( 2 ) M. Nitta, T. Yachi, and T. Tani: "Experimental study for wind powerhydrogen energy system with energy capacitor system", IEICE/IEEE INTELEC'03, pp.451-456 (2003)

( 3 ) H. Kaneuchi, M. Nitta, T. Yachi, and T. Tani: "Scale Estimation of a Wind Turbine System with EDLC for Hydrogen Production", IEICE/IEEE Technical Committee on Energy Engineering in Electronics and Communication'04, pp.7-11 (2004) (in Japanese)

( 4 ) H. Kaneuchi, M. Nitta, T. Yachi, and T. Tani: "The optimum EDLC capacity of a Wind Turbine System for Hydrogen Production", Solar/Wind Power Energy Lecture Collected Papers, pp.337-340 (2004) (in Japanese)

( 5 ) M. Okamura: "Electric double layer capacitor and accumulation-ofelectricity system", Nikkan Kogyo Shimbun (1999) (in Japanese)

( 6 ) http://www.windenergy.com

( 7 ) http://www.jma.go.jp/JMA_HP/jma/indexe.html

( 8 ) NEDO: A wind power introduction guidebook (2001) (in Japanese) 
Hironobu Kaneuchi (Student Member) was born in Tokyo, Japan. He received a B.S. degree from the Faculty of Engineering at the Tokyo University of Science. He is a graduate student at the Tokyo University of Science. He studies hydrogen producing system and is a member of the Japan Solar Energy Society (JSES).

Toshiaki Yachi (Member) received B.S. and M.S. degrees from the

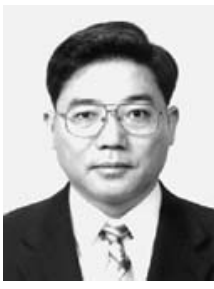

Faculty of Engineering at Shizuoka University, Japan, in 1973 and 1975, respectively, and a Dr. degree of Engineering from the Graduate School of Electronic Science and Technology, Shizuoka University in 1986. He joined Nippon Telegraph and Telephone Corporation (NTT) Electrical Communications Laboratories, Tokyo, Japan in 1975. In 2002, he was appointed to the Faculty of Engineering of the Tokyo University of Science as a Professor. $\mathrm{He}$ is currently engaged in research on power devices, power supplies, photovoltaic systems and fuel cell systems. He served as Program Committee Chairman of the 1998 International Symposium Power Semiconductor Devices \& ICs (ISPSD'98), Steering Committee Chairman of ISPSD'01, and General Chairman of ISPSD'04. He also served as Program Committee Chairman of the 2003 International Telecommunication Energy Conference (INTELEC'03). He is a member of the Institute of Electrics, Information, and Communication Engineers of Japan, the Japan Society of Applied Physics, the Japan Solar Energy Society, and the IEEE (Fellow).
Tatsuo Tani (Member) received his Ph.D. from Nagoya University in 1980. He joined the Electro Technical Laboratory of the Ministry of International Trade and Industry in April 1966. After serving as Head of the Solar Energy Research Section of Energy Division at Laboratory, he was appointed as Professor, the Faculty of Engineering, Tokyo University of Science in April, 1988 and has been at the Faculty of System Engineering, TUS, Suwa since April, 2002, and is involved with Energy Conversion Engineering. He is a member of the International Solar Energy Society. 